\title{
Comparing Stress ECG Enhancement Algorithms
}

\section{With an introduction to a filter bank based approach}

There are two predominant types of noise that contaminate the electrocardiogram (ECG) acquired during a stress test: the baseline wander noise (BW) and electrode motion artifact, and electromyogram-induced noise (EMG) [1]. BW noise is at a lower frequency, caused by respiration and motion of the subject or the leads. The frequency components of $\mathrm{BW}$ noise are usually below $0.5 \mathrm{~Hz}$, and extend into the frequency range of the ST segment during a stress test. EMG noise, on the other hand, is predominantly at higher frequencies, caused by increased muscle activity and by mechanical forces acting on the electrodes. The frequency spectrum of the EMG noise overlaps that of the ECG signal and extends even higher in the frequency domain. In this article, we review some of the published ECG enhancing techniques to overcome the noise problems, and compare their performance on stress ECG signals under adverse noise scenarios. We also describe the Filter Bank (FB) based ECG enhancing algorithm [9].

\section{Overview}

Figure 1 shows a noise-free ECG beat with ST-segment depression induced by exercise (top) and various epochs of this ECG with different noise conditions, as during a stress test. It is important to measure the dynamic changes in the morphology of segments of the ECG induced by the exercise, even in the presence of noise.

Many ECG enhancing techniques to address the noise problem have been reported in the literature. In Ref. [2] a combination of mean and median algorithms is used on the filtered ECG. Reference [3] presents a BW noise removal filter which meets specifications in Ref. [4] and a timevarying filter to remove high-frequency EMG noise. Reference [5] provides a technique which subtracts the current heart-beat average to get a 'QRS-free' signal, estimates the BW from the downsampled QRS-free signal, and then sub-

May/June 1996

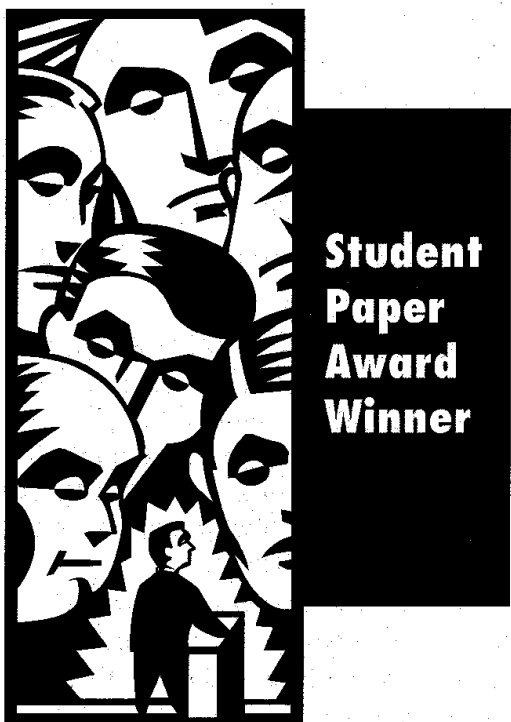

tracts the estimated BW from the noisy ECG. Reference [6] uses a source consistency filtering technique that seeks to develop a transfer function of the cardiac dipole. In Ref. [7], an adaptive baseline wander filter is designed as a cascade of two adaptive filters. Reference [8] uses a cubic spline technique to estimate and then subtract the BW in the ECG.

The cubic spline method works well

Valtino X. Afonso
Department of Electrical and Computer Engineering
University of Wisconsin
Willis J. Tompkins
Department of Electrical and Computer Engineering
University of Wisconsin
Truong Q. Nguyen
Department of Electrical and Computer Engineering
University of Wisconsin
Kurt Michler
Burdick Inc.
Shen Luo
Burdick Inc.

when the 'knots' used to estimate the BW are accurately determined. However, the determination of the knots can be adversely affected by a noisy ECG signal, which compromises the $\mathrm{BW}$ estimate. Adaptive filtering of the ECG assumes that either the signal or noise is stationary or nonstationary. These characteristics are not guaranteed in a stress ECG recording.

The various ECG enhancement algorithms addressed in this article compute an enhanced beat from a set of ECG beats or epochs. We refer to the enhanced beat of any enhancing algorithm as the composite beat. Other articles may refer to the enhanced beat as the "averaged' beat, but we wish to avoid confusion with the usual sense of the word "averaged," which indicates the arithmetic mean of data.

\section{Signal Enhancing Algorithms}

1. Mean composite: The mean composite $\mathbf{C}_{\text {mean }}$ is determined by computing the arithmetic mean of a set of noisy beat epochs. The epochs are time aligned using a fiducial point in the heart beat, such as the $R$ wave. The mean composite is given by:

$$
\begin{aligned}
& C_{\text {mean }}(n)=\frac{1}{N} \sum_{k=1}^{N} x_{k}(n) \\
& 0 \leq n \leq L-1
\end{aligned}
$$

where $\mathbf{x}_{\mathrm{k}}, k=1, \ldots, \mathrm{N}$ are the noisy epochs of length $L$.

This is the simplest strategy of enhancing the ECG. In an ideal situation, where the noise is uncorrelated with the signal, is stationary, and has a Gaussian distribution, the signal-to-noise-ratio is improved by a factor of $\sqrt{N}$.

If one of the epochs has a sudden ECG baseline shift, or is an arrhythmia such as a ventricular ectopic beat, the resulting mean composite will be distorted. A preprocessing step prior to the mean composite algorithm should determine the 'goodness' of the epoch and decide 
whether it should be included in the composite or rejected.

2. Median composite: The nedian composite, $\boldsymbol{C}_{\text {median }}$, is determined by computing the median of sample values across the set of epochs, for each time instant. The epochs are time aligned by using a fiducial point in the heart beat, such as the $\mathrm{R}$ wave.

$C_{\text {median }}(n)=\operatorname{median}\left\{x_{1}(n), x_{2}(n), \ldots, x_{N}(n)\right\}$

$0 \leq n \leq L-1$

where $\mathbf{x}_{\mathrm{k}}, k=1, \ldots, \mathrm{N}$ are the noisy epochs of length $\mathrm{L}$.

The median technique remōyes any outliers in the distribution of data at each instant across the epochs. Thus, baseline shifts in an epoch will be disregarded and not affect the median composite. Bursts of high frequency noise in the ECG will be removed, since these represent outliers in the set of epochs to be composed.

3. Hybrid composite: The hybrid composite algorithm combines the benefits of the mean and median composite algorithms [2]. The mean composite algorithm is computationally efficient, and optimal for high-frequency noise reduction, but is susceptible to low-frequency noise. The median composite algorithm is computationally expensive. Their combination is an algorithm that is near optimal for high-frequency noise, good at low-frequency noise reduction, and is computationally efficient.

In the hybird algorithm, a sequence of incoming beats is partitioned into three groups by using one of three strategies. A random grouping strategy partitions the incoming beats into the three groups randomly. A block grouping strategy partitions the first one-third of the epochs into the first group, the second one-third into the second group, and the final one-third into the last group. A sequential grouping strategy partitions sequential beats into different groups. The arithmetic mean composite is computed for each of the three groups, regardless of the strategy used. The baseline level estimated from the $P Q$ segment is then removed from each of the three mean composites. For each group, a low-pass filter with a comer frequency of approximately $15 \mathrm{~Hz}$ is used to remove the correlation between the high-frequency noise and the low-frequency noise:

The hybrid composite algorithm is then obtained by summing the median of the low-frequency signals with the me- dian of the high-frequency signals. The algorithm is represented as follows:

$$
\begin{gathered}
\mathrm{E}=\left\{\mathrm{x}_{1}, \mathrm{x}_{2}, \ldots, \mathrm{x}_{N}\right\} \\
\mathrm{E}_{k}=\operatorname{Group}(\mathrm{E}) \\
\mathrm{y}_{k}=\text { mean_composite }\left(\mathbf{E}_{k}\right) \quad k=1,2,3 \\
\mathrm{z}_{k} \text { baseline_correction }\left(\mathrm{y}_{k}\right) \quad k=1,2,3
\end{gathered}
$$

$L P_{k}(n)=$

$\frac{1}{J}\left(z_{k}\left(n-\frac{J}{2}\right)+z_{k}\left(n-\frac{J}{2}+1\right)+\ldots+z_{k}\left(n+\frac{J}{2}\right)\right)$

$$
\begin{array}{r}
k=1,2,3 \\
H P_{k}(n)=z_{k}(n)-L P_{k}(n) \quad r \\
k=1,2,3 \\
\mathrm{C}_{H y b}=\text { medtan_composite }\left\{\mathrm{LP}_{1}, \mathrm{LP}_{2}, \mathrm{LP}_{3}\right\}+ \\
\text { median_composite } \left.\mathrm{HP}_{1}, \mathrm{HP}_{2}, \mathrm{HP}_{3}\right\}
\end{array}
$$

where $\mathbf{x}_{1}, \mathbf{x}_{2}, \ldots, \mathbf{x}_{\mathrm{N}}$, are the noisy epochs, $N$ is the number of noisy epochs each of length $\mathbf{L}$, and $\mathbf{J}$ is chosen based on the sampling frequency to get a cutoff frequency of approximately $15 \mathrm{~Hz}$. LP and IIP refer to the low-passed and highpassed signals respectively.

4. Trimmed mean composite: $A$ trimmed composite $\mathrm{C}_{\text {rrim }}$ of a set of timealigned epochs is computed by first sorting the values at each time instant through the epochs. The bottom $20 \%$ and top $20 \%$ of the 'sorted' epochs are discarded. For example, if there are 10 epochs in an episode, after sorting and discarding there will be $10-2-2=6$ "sorted" epochs left. The arithmetic mean of the remaining epochs is then computed to obtain the trimmed composite:

$$
\begin{gathered}
\mathbf{E}=\left\{\mathbf{x}_{1}, \mathbf{x}_{2}, \ldots, \mathbf{x}_{N}\right\} \\
\mathbf{E}^{\prime}=\operatorname{sort}(\mathbf{E})=\left\{\dot{\mathbf{x}}_{1}^{\prime}, \mathbf{x}_{2}^{\prime}, \ldots, \mathbf{x}_{N}^{\prime}\right\}
\end{gathered}
$$

where

$$
\begin{gathered}
x_{1}^{\prime}(n) \leq x_{2}^{\prime}(n) \leq \ldots \leq x_{N}^{\prime}(n) \quad 0 \leq n \leq L-1 \\
\mathbf{E}^{\prime \prime}=\left\{\mathbf{x}_{i+1}^{\prime}, \ldots, \mathbf{x}_{N-i}^{\prime}\right\} \quad i=\operatorname{round}(0.2 \times N) \\
\mathbf{C}_{\text {Trim }}=\text { mean composite }\left(\mathbf{E}^{\prime \prime}\right)
\end{gathered}
$$

and where $\mathbf{x}_{1}, \mathbf{x}_{2}, \ldots, \mathbf{x}_{\mathrm{N}}$, are the noisy epochs, $\mathbf{x}^{\prime}{ }_{1}, \mathbf{x}^{\prime}{ }_{2}, \ldots, \mathbf{x}^{\prime} \mathrm{N}$, are the "sorted" noisy epochs, and $\mathrm{N}$ is the number of noisy epochs each of length L. E' is the set of "sorted" noisy epochs, and $\mathbf{E}$ " the trimmed set of "sorted" noisy epochs.

The trimmed mean algorithm removes outlier data values in the set of epochs to be composed before computing the mean composite of the residual data. It thus incorporates a feature of the median algo rithm, in that extreme data values, or outHers $s$ do not influence the resultant composite In addition, the use of the mean algorithm to determine the final compesite ensures optimal noise reduction in the case of Gaussian distributed, uncorrelated, and stationary noise.

5. Incremental composite: The fixed increnental composite, $\mathbf{C}_{\text {IncrF }}$ is computed by increasing or decreasing each sample value in the current composite beat by a fixed amount [10]. The direction of the change in value of each sample de pends on the corresponding sample in the next epoch. If the sample in the noise epoch is greater than the corresponding one in the current (running) ineremental beat, the latter point is increased by afixed amount. The increment parameter in the algorithm is specified as corresponding to a specifie $m m$ distance on a strip chart recording of the ECG with a $1 \mathrm{mV} / \mathrm{cm}$ vertical axis sensitivity. This choice provides a balance between mmunity to noise and dynamic response of the composite.

The algorithm for the fixed ineremental composite is given as:

$$
\begin{aligned}
& \mathrm{E}=\left\{\mathrm{x}_{1}, \mathrm{x}_{2}, \ldots, \mathrm{x}_{N}\right\} \\
& \mathrm{C}_{\text {Incir } F}=\mathrm{x}_{1} \\
& \text { for } k=2 \text { toN } \\
& \Delta=\mathrm{C}_{\text {ING } \mathrm{C}_{\mathrm{K}}}-\mathrm{X} \\
& \mathrm{C}_{\text {IncrF }}=\mathrm{C}_{\text {Ih }{ }^{2} F}-\mathrm{P}_{\text {Fired }} \times \operatorname{sigh}(\Delta) \\
& 3
\end{aligned}
$$

where $\mathbf{x}_{1}, \mathbf{x}_{2}, \ldots, \mathbf{x}_{\mathrm{N}}$, are the noisy epochs, sign $(\Delta)$ operates on each element of the vector $\Delta$, and is 1 if $\Delta(\mathrm{n})>0,-1$ if $\Delta(\mathrm{n})<$ 0, or 0 otherwise, $N$ is the number of noisy epochs each of length L, and $P_{\text {Fixed }}$ is the fixed incremental parameter.

A second version of this algorithm computes a percentage incremental composite $\mathbf{C}_{n c r}$, where the incremental parameter is a pereentage of the difference between the running incremental beat and the incoming epoch.

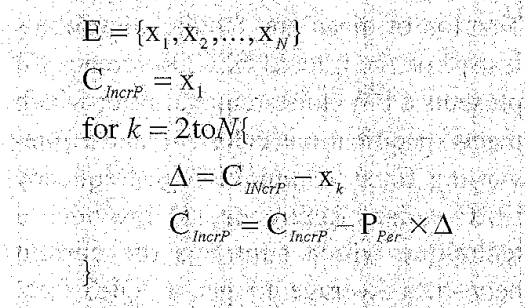

where $\mathbf{x}_{1}, \mathbf{x}_{2}, \ldots, \mathbf{x}_{\mathrm{N}}$, are the noisy epochs, $\mathrm{N}$ is the number of noisy epoehs each of 
length $\mathrm{L}$, and $\mathrm{P}_{\mathrm{Per}}$ is the percentage incremental parameter.

\section{Filter Bank Based Composite}

The filter bank-based strategy relies on a filter bank (FB) containing a set of analysis filters $H_{l}(z)$ and synthesis filters $F_{l}(z)$ (Fig. 2). The analysis filters decompose the input signal, $x(n)$, into $\mathrm{M}$ frequency bands (subbands) and subsample by a factor of M. Processing can be performed on each subband independently. The synthesis filters combine the processed subbands to reconstruct the input signal. Thus, a FB-based algorithm involves decomposing a signal into frequency subbands, processing these subbands according to the application, and reconstructing the processed subbands. The design and use of FBs is widely reported in the literature [11-13].

Many signals contain specific energy distributions in the frequency domain. For example, a significant proportion of the energy from the QRS complex in the ECG extends to a frequency of $40 \mathrm{~Hz}$, and even more if the $\mathrm{Q}, \mathrm{R}$, and $\mathrm{S}$ waves have a very sharp morphology. The $P$ and $T$ waves, in general, have a significant proportion of energy only up to $10 \mathrm{~Hz}$. Thus, there is a benefit in using a FB-based algorithm, where time and frequency dependent processing can be performed.

FB block diagram: Assume that the FB contains $M$ analysis and synthesis filters, each of length $\mathrm{L}$. The analysis filters $H_{I}(z)$, $l=0,1, \ldots, \mathrm{M}-1$, bandpass the input signal $X(z)$ to produce the subband signals $U_{l}(z)$ :

$$
U_{l}(z)=H_{l}(z) X(z) \quad l=1,2, \ldots, M-1
$$

Since the effective bandwidth of $U_{l}(z)$ is $\frac{2 \pi}{M}$, it can be downsampled to reduce the total rate. The downsampling process keeps one sample out of $\mathrm{M}$ samples. The downsampled signal $W_{l}(z)$ is:

$$
\begin{aligned}
& W_{l}(z)=\frac{1}{M} \sum_{k=0}^{M-1} U_{l}\left(z^{1 / M} e^{-j k 2 \pi / M}\right) \\
& \text { for } l=0,1, \ldots M-1
\end{aligned}
$$

Taking advantage of the downsampling, we can efficiently apply the filtering process at $\frac{1}{M}$ the input rate. This implementation is referred to as polyphase implementation and contributes to the computational efficiency of the FB-based algorithm [11].

Time and frequency dependent processing can now be performed on some or all of the subband signals to result in a

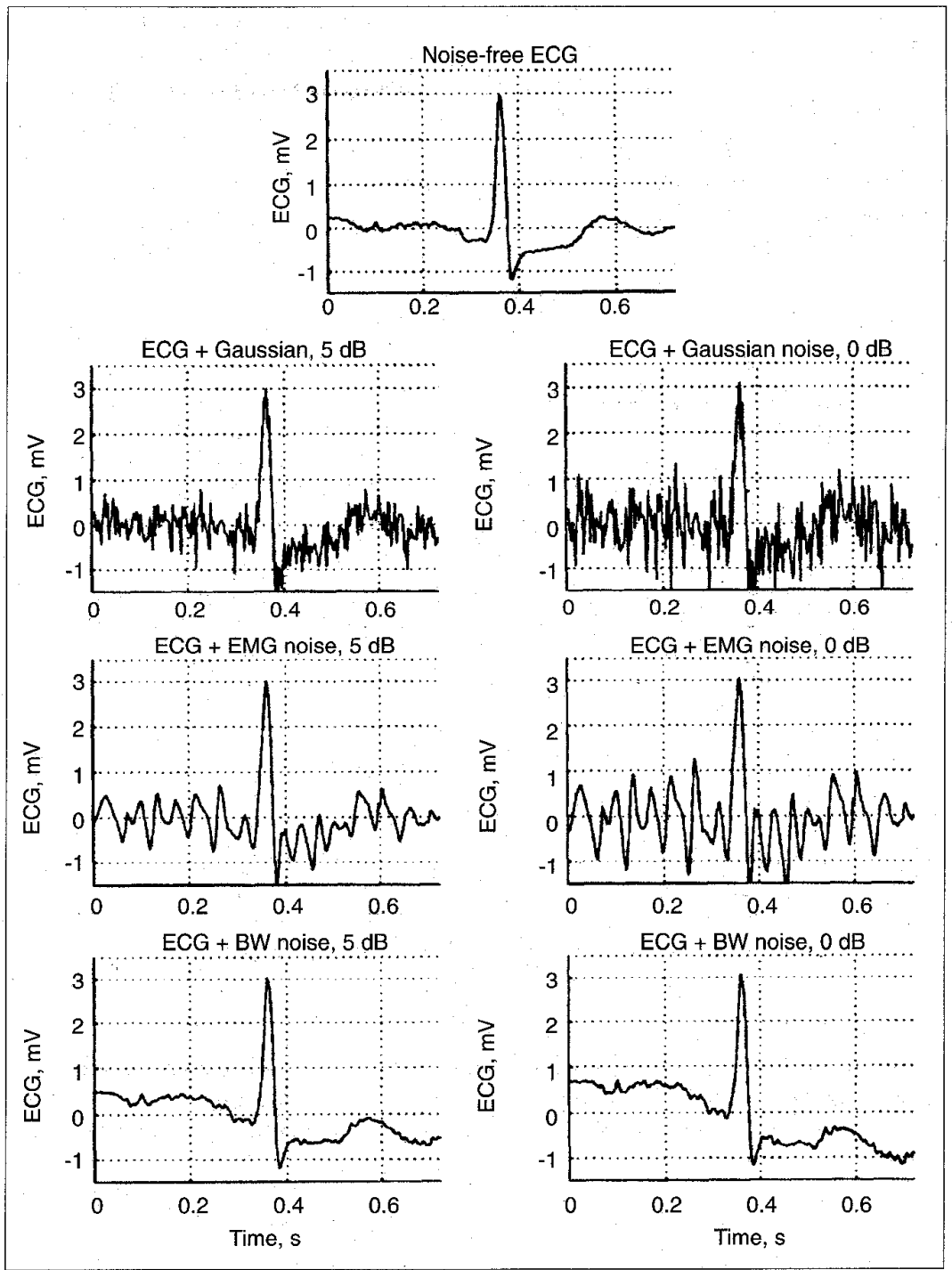

1. Examples of a noise-free ECG beat contaminated with various types of noise. EMG is electromyogram noise and BW is baseline wander noise. The SNR level is low enough so as to distort the underlying ECG signal.

processed and downsampled subband signal, $W p l(z)$. The nature of the processing involved is application dependent, and stress ECG enhancement is explained later.

The reconstruction is achieved by upsampling and interpolating the subband signals using a set of bandpass filters, $F_{l}(z)$. Similar to the analysis bank, the filtering process in the synthesis bank can be efficiently implemented by taking advantage of the M-1 zeros in the upsampled sequence, $V_{l}(z)$. This processing contributes to the overall computational efficiency of the FB-based algorithm.

The processed subband signals, $O_{l}(n)$, can then be added algebraically point-by- point to result in a time and frequency dependent processed version, $Y(z)$, of the input signal, $X(z)$. The analysis and synthesis filters must be designed to incorporate useful properties for the application at hand.

Filter Bank Properties

The analysis filters decompose the ECG into uniform frequency subbands. For stress ECG enhancement, and many other ECG processing tasks, it is useful while processing each subband to have a fixed or deterministic relationship between data in the processed subbands and data at the input. This requirement implies that the analysis filters have linear phase frequency characteristics, or constant 
group delay for all frequencies in the passband.

The linear phase requirement ensures that all frequencies at the input will have the same sample delay through the analysis filters. It is then possible, for example, to determine the exact location of the $\mathrm{R}$ wave and other fiducial points in the sub-

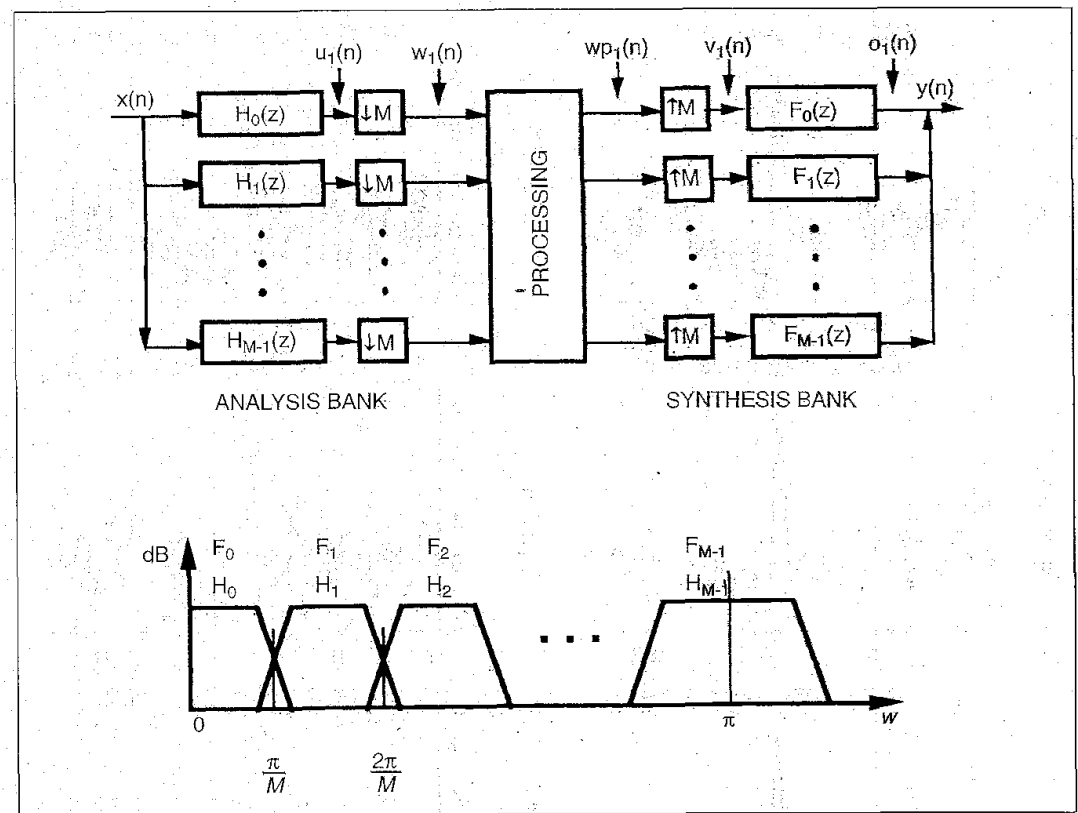

2. A filter bank-based algorithm enables time and frequency dependent processing with a reduced number of computations per second. Ideal magnitude responses of the filters are shown. One set of filters has the potential to accomplish multiple ECG processing tasks.

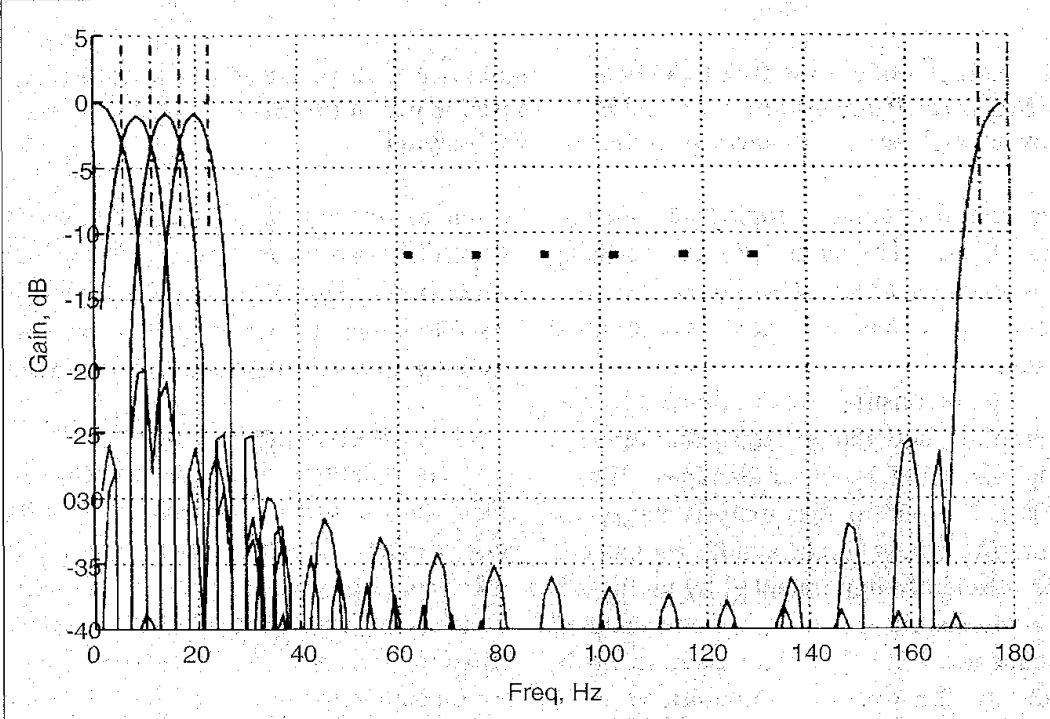

3. The magnitude responses of the 32 channel filter bank. These filters have linear phase, are orthogonal, and have the perfect reconstruction property. downsampling, due to the nonideal nature of the analysis and synthesis filters. Secondly, the output $y(n)$, could have a magnitude distortion compared to the input, $x(n)$, if the analysis and synthesis filters were not designed to cancel effeets due to the nonideal magnitude response of the filters. Finally, if the output cannot be represented in terms of the input by a simple sample delay it couldhave a phase distortion compared to the input. Thus, it is possible for the FB system output to have no phase distortion with respect to the input (ie, a linear phase FB) even if each of the analysis and synthesis filters have nonlinear phase frequency responses In ECG processing however, it is important that each of the analysis and synthesis filters have a linear phase frequency response.

\section{Perfect Reconstruction}

The output of the FB can differ from the input due to the yarious reasons stated above. The processing block incorporated into the FB system will itself cause the output to differ from the input The FB used in this study is a perfect reconstruction ( $\mathrm{PR}$ ) system that reproduces the input signal at the output when there is no processing The ideal relationship between the input and output in Fig. 2 is:

$$
y(n)=c x(n-k)
$$

where $k$ is the system delay, and $c$ is a constant gain factor.

Aliasing and inaging distortions are the results of nenideal magnitude responses of the filters. The decimated signal in Fig. $2, W_{l}(z)$, has aliased frequencies of the input. These errors can be cancelled if one designs the analysis and synthesis filters appropriately. This is $\mathrm{Nell}$ explained in various sources in the literature $[11-13]$.

In an alias free $\mathrm{FB}, y(z)-T(z) \mathrm{X}(z)$, where $T(z)$ is the overall distortion function. Clearly, there is no distortion if $T(z)$. $=z^{-k}$. The system has no magnitude distortion if $T(z)$ is an all pass function; and there is no phase distortion if $T(z)$ is a linear phase function. PR requires that the overall transfer function of the FB have an all-pass magnitude response ( $c$ is a constant), and a linear phase response ( $k$ is a fixed delay).

The reason for using the PR property of filter banks is that an overall goal is to develop one set of filters which will work well for various ECG processing tasks. Neither ECG beat detection nor classifica 
tion requires reconstruction at the output of the filter bank (see Fig. 2). For these applications, only decomposition of the input into frequency subbands is of interest. Stress ECG enhancement, however, requires reconstruction of the processed subbands. This task requires a PR filter bank with no phase or magnitude distortion.

The 32-channel FB was designed, based on an algorithm from Ref. [14]. The analysis and synthesis filters have linear phase responses and their magnitude responses are as shown in Fig. 3.

\section{Subband Processing}

The FB structure enables processing of a signal in a specific time period and a specific frequency region. The FB-based algorithm decomposes the ECG into 32 uniform subband frequencies of the signal (see Fig. 3). The 0 to $180 \mathrm{~Hz}$ frequency bandwidth of the input signal is decomposed into 32 uniform frequency subbands, $\{[0$ to 5.625$],[5.625$ to 11.25$], \ldots$, [174.375 to 180$]\} \mathrm{Hz}$.

The subband in the [0 to 5.625$] \mathrm{Hz}$ range, which contains most of the energy of the $\mathrm{P}$ and $\mathrm{T}$ waves, is not processed in any way. In the remaining subbands, the signal components are attenuated to various levels in time periods that correspond to the non-QRS region. Since the $\mathrm{P}$ and $\mathrm{T}$ waves do not have energy at higher frequencies, we attenuate the signal components in these time periods and frequencies. The QRS region of the ECG is not modified in any of the subbands. The processed subband signals are then reconstructed by the synthesis filters to result in a time and frequency dependent processed version of the input signal, in which noise has been reduced without distorting ECG components of interest.

The $Q$ and $S$ fiducial points to mark the QRS complex are determined from the input noisy ECG using the algorithm described in Ref. [15]. Since each of the analysis filters has linear phase, the $Q$ and $S$ waves can be located in each subband signal after accounting for filter delays.

\section{FB-based Composite}

An FB based composite is obtained by performing the above operations on the input ECG. To compute a FB based composite from a set of more than one noisy epoch, the FB composite is computed for each epoch, and then the mean of the resulting composites computed.

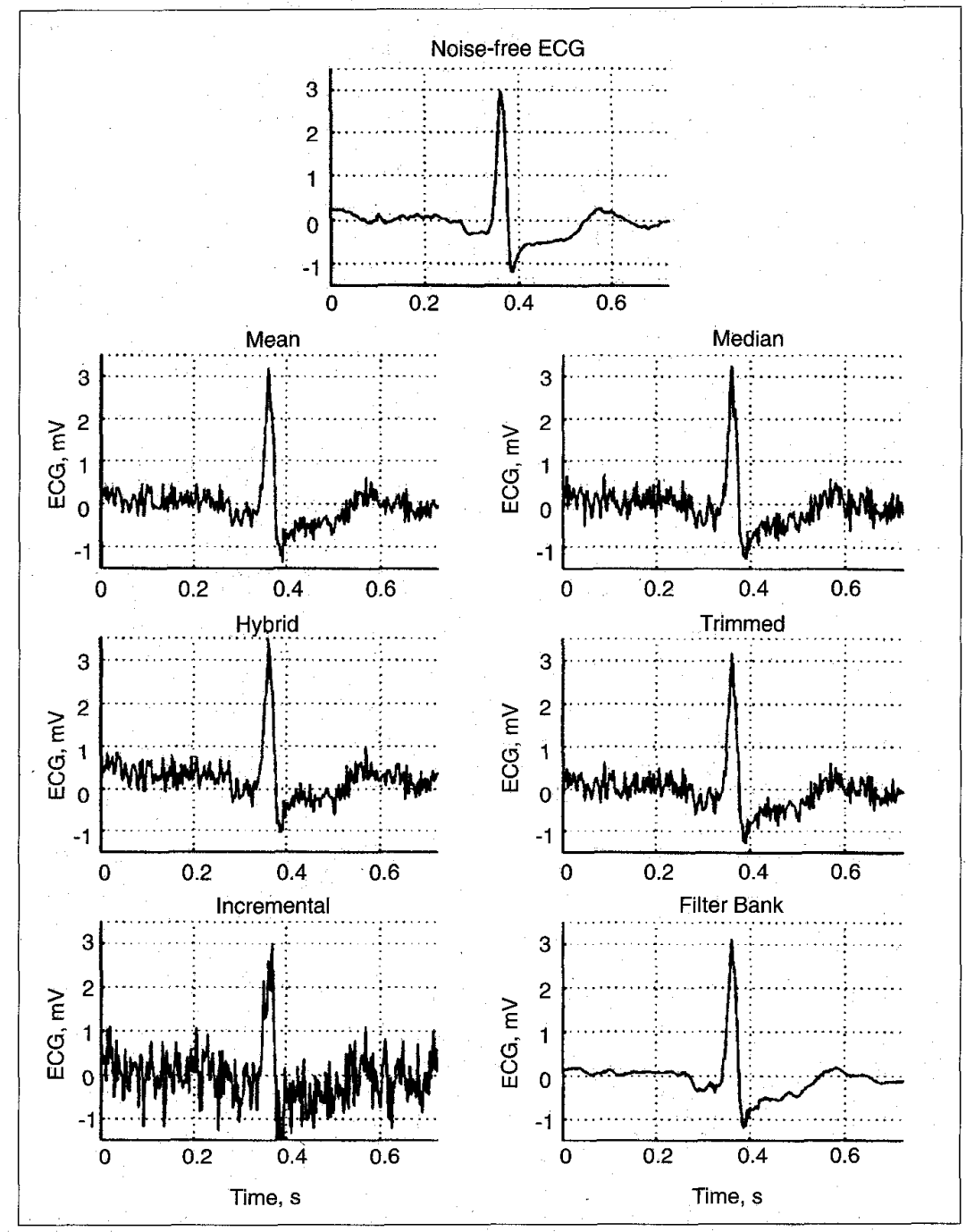

4. Composites from various enhancing algorithms using nine noisy epochs of the noise-free ECG. Under a Gaussian noise scenario, $(\mathrm{SNR}=0 \mathrm{~dB})$, the FB-based composite has the closest resemblance to the noise-free ECG than the other composites.

\section{Methods}

\section{Stress ECG data}

A noise-free ECG beat cycle with an exercise-induced ST segment depression was obtained from the MIT/BIH database [16]. This beat was used as a template of a noise-free ECG epoch. Noisy beat epochs were constructed by adding purenoise segments to the template. Noisy data were either generated artificially or obtained from the MIT/BIH database. Artificial noisy data were generated with an independent Gaussian distribution. BW and EMG noise were read from the MIT/BIH database [1]. The noisy beat epochs could be accurately aligned, since the location of the $\mathrm{R}$ wave was known from the underlying noise-free ECG beat.
Signal-to-noise-ratio: We used the SNR parameter to quantify and compare the performance of the algorithms, and also to determine the noise level in an enhanced ECG beat. The SNR was defined as [17]:

$$
S N R=10 \times \log _{10} \frac{S_{\sigma}}{N_{\sigma}}
$$

where $\mathbf{S}$ was a noise-free $\mathrm{ECG}$ template of length $L, N$ was a noise vector of length $\mathrm{L}$, and $\mathrm{X}_{\sigma}$ was defined as:

$$
X_{\sigma}=\sum_{l=0}^{L-1}\left(X(l)-\mu_{\mathrm{x}}\right)^{2}
$$

where $\mu \mathbf{x}$ was the mean of the signal $\mathbf{X}$.

To generate a noisy epoch of an ECG beat, the sample values of the noise vector 


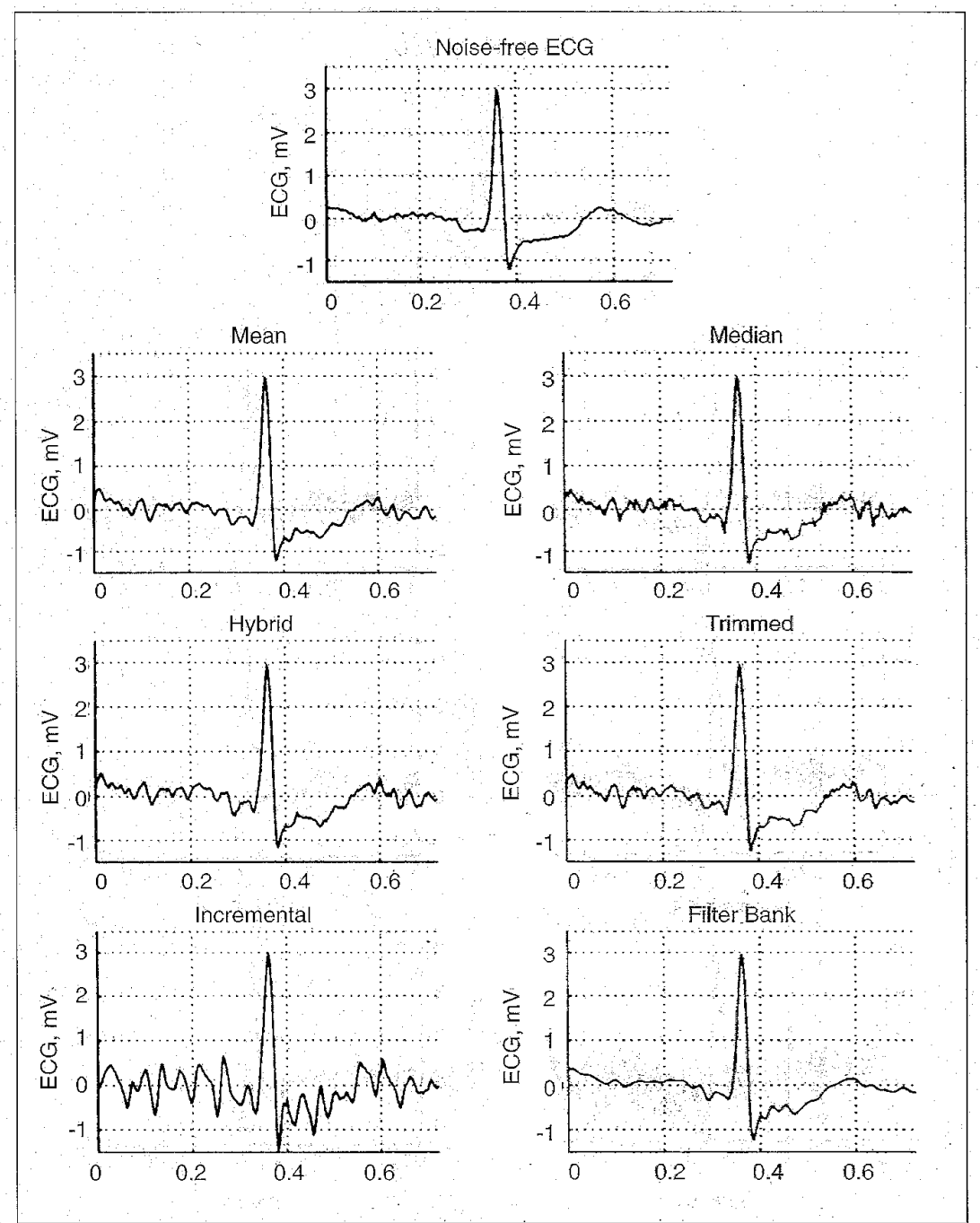

5. Composites from various enhancing algorithms using nine noisy epochs of the noise-free ECG. Under a EMG noise scenario, $(\mathrm{SNR}=5 \mathrm{~dB})$, the FB-based composite has a good resemblance to the noise-free ECG compared to the other composites.

were multiplied by a gain factor corresponding to the required SNR and added to the noise-free ECG beat. To compute the SNR of an ECG composite beat, the known noise-free ECG beat was subtracted to determine the residual noise; and then the above SNR formula was used.

\section{ECG Composites}

Figure 1 shows sample noisy stress ECG epochs under various noise scenarios. We computed the composite of each of the algorithms when input with nine noisy ECG epochs. Each of the nine epochs had the same underlying noise-free ECG beat. The Gaussian $(0 \mathrm{~dB})$, and EMG $(5 \mathrm{~dB})$ noise scenarios were tested. Based on initial experiments, we decided to use the fixed increment method.

\section{SNR Improvement}

For each algorithm, we computed the SNR of the composite beat from $1,2,3$, .., 9 epochs. Based on this experiment, a comparison can be made among algorithms to determine which results in an increased SNR with fewer epochs: Since the hybrid algorithm partitions the set of noisy epochs into three groups, the incremental numbers of epochs used for this particular algorithm were three, six, and nine. The trimmed method works on a reduced set of the input epochs Thus, in this experiment, this method only begins to show SNR improvement after about five epochs:
Results

\section{Stress ECG data}

Figure 1 shows samples of noisy ECGs Each of the noisy epochs used is very distorted as compared to the noisefree beat, so as to test the robustnes of the algorithms.

\section{ECG Composites}

Figure 4 shows the output of each al gorithm using nine epochs with Gaussian distributed noise and a SNR of $0 \mathrm{~dB} F \mathrm{Fig}$ ure 5 shows the output of each algerithm using nine epochs with EMG distributed noise and a SNR of $5 \mathrm{~dB}$. Under both noise seenarios, the $\mathrm{FB}$ based composite shows best resemblance to the noise-free beat The $P$ and $T$ waves are closest in morphol ogy to the noise-free beat in the ease of the FB composite, The mean algorithm has a favorable performance under EMG noise. The other composites do show a re due tion in noiselevel as compared to ther original epochs (see Fig. 1)

\section{SNR Improvement}

Figure 6 shows the SNR uiprovenent as a fanction of the number of epochs included in the composite. The FB based method shows the best SNR improvement under Gaussian and EMG noise scenarios. The mean, median, hybrid, and trimmed methods have similar performance. The incremental algorithm does not show as remarkable a SNR improvement as do the other algorithms.

The FB based composite has a bigher SNR improvement using a fewer number of epochs than the other techniques For example, in the EMG noise scenario (5 dB), the FB composite of four epochs has a SNR improvement of about $15 \mathrm{~dB}$, as compared to the mean algerithm which attains $15 \mathrm{~dB}$ only after nine epochs (see Fig. 6 ,

\section{Discussion and Summary}

We used stress ECG data that is extremely contaminated with noise because it was important to test and present results of stress ECG enhaneing algoritbins operating under adverse noise scenarios.

In a stresS ECG system, the entianced beat is further processed to measure parameters from the ST segment and the overall ECG morphology It is thus important that the enhanced beat be as noise free as possible, since noise hampers further processing. For example, computing the ST segment depression or elevation re- 
quires determining the isoelectric level as well as the detection of the $J$ point and the onset of the $T$ wave. These processing tasks are compromised by too high a noise level in the enhanced ECG.

The mean algorithm operates best in a stationary and uncorrelated noise scenario. These ideal scenarios do not usually exist in stress ECG records. The median algorithm works well at removing extreme data values in a distribution of ECG epochs. However, a usual preprocessing step for an ECG enhancing algorithm determines the 'goodness' of an ECG epoch before it is included in the set of epochs to be composed. Thus, this primary benefit of the median algorithm normally occurs of in this preprocessing step.

The hybrid algorithm removes noise fairly well as compared to the mean composite, but in addition better handles baseline shifts present in epochs than does the mean algorithm [2]. The trimmed mean method inherently combines mean and median techniques. The incremental algorithm does not directly exploit the nature of the noise. Thus, its enhancing performance is minimal. However, in a noise-free scenario, the incremental technique is potentially useful to track quickly and accurately dynamic changes in the ST segment as compared to the mean composite algorithm, which averages dynamic ST segment changes. Its usefulness in this area remains to be studied.

The FB composite enhances the ECG best under both Gaussian and EMG noise scenarios, and is also computationally inexpensive. It operates on the noise characteristics in the time and frequency domains, independently. The FB also potentially offers a way to perform other ECG processing tasks, such as $R$ wave detection, enhancement and beat classification. These tasks can be carried out because the analysis filters decompose the ECG into various subbands, which can be used for further analysis. Thus, with one set of filters, various tasks could be performed in parallel, improving the overall computational efficiency of existing ECG processing systems.

As for improving the SNR, during a stress test it is important to measure changes in the ST segment as quickly as possible after they occur. Most enhancing algorithms require a set of ECG epochs from which one enhanced beat is computed. Various features of the morphology (such as ST segment depression) are then computed from this enhanced beat by us-

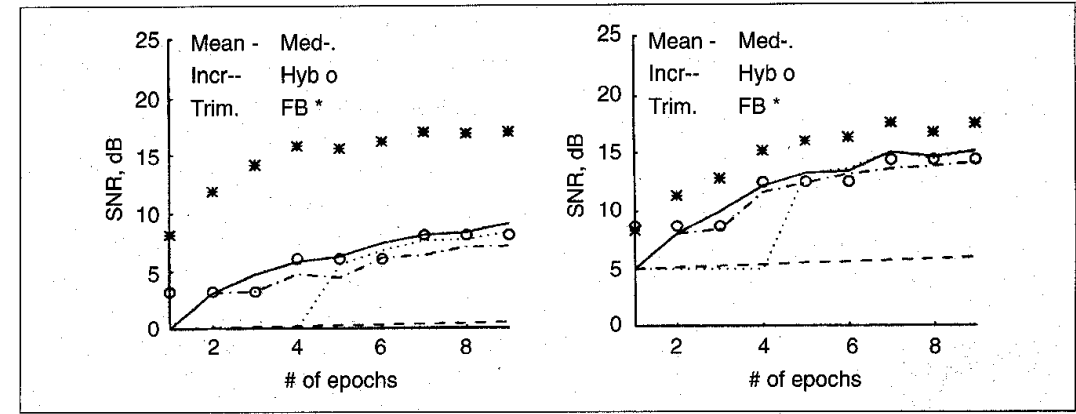

6. SNR improvement over an incremental number of epochs under Gaussian (left) and EMG noise scenarios. The FB composite shows better SNR improvement with a fewer number of epochs

ing other algorithms. Using a large number of epochs in the set will result in 'time-averaged' features. It is thus important to get an enhanced ECG using as few epochs as possible.

The FB-based composite provides the best SNR in the fewest number of beats. However, studies still remain to be performed on the accuracy of ST measurements under various noise scenarios. For example, in a noise-free scenario it is likely that the incremental algorithm will track changes in the ST segment more quickly and accurately than the mean algorithm.

We also compared the performance of the algorithms on ECGs contaminated with Gaussian and EMG noise only. A high-pass filter such as the one described in Ref. [3] or any other that meets the specifications given in Ref. [4] can be used to preprocess the ECG before using any of the above algorithms. In fact, the high-pass filter can be incorporated into the lowest subband of the FB algorithm. This filter would then operate at a lower rate since it is filtering the downsampled 0 to $5.625 \mathrm{~Hz}$ subband. The resulting FB algorithm would then filter BW and EMG noise.

\section{Acknowledgments}

This work was supported by a grant from Burdick Inc., Milton, WI., USA. The authors would like to thank Steffen Trautmann for his help with the design of the filter banks.

\section{References}

1. Moody GB, Muldrow WK, and Mark RG: A noise stress test for arrhythmia detectors. Computers in Cardiology. pp. 381-384, 1984.

2. Mertens $\mathbf{J}$ and Mortara D: A new algorithm for QRS averaging. Comp. in Cardiol., pp. 367-369, 1984

3. Pinto V: Filters for the reduction of baseline wander and muscle artifact in the ECG. Journal of Electrocardiology., vol. 25, suppl., pp. 40-48, 1992.

4. Bailey JJ, Berson AS, Garson A, Horan LG, Macfarlane PW, Mortara DW, and Zywietz C: Recommendations for the standardization and specifications in automated electrocardiography: Bandwidth and digital signal processing. A report for health professionals by an ad hoc writing group diac electrophysiology of the council on clinical cardiology, American Heart Association. Circulation. no. 2, pp. 730-739, 1990

5. Sornmo L: Time-varying filtering for removal of baseline wander in exercise ECGs. Computers in Cardiology. pp. 145-148, 1991.

6. Mortara DW: Source consistency filtering -A new tool for ECG noise reduction. Computers in Cardiology. pp. 125-128, 1991.

7. Jane R, Laguna P, Thakor NV, and Caminal P: Adaptive baseline wander removal in the ECG: Comparative analysis with cubic spline technique. Computers in Cardiology., pp. 143-146, 1992.

8. Meyer, CR, and Keiser HN: Electrocardiogram baseline noise estimation and removal using cubic splines and state-space computation techniques. Computers and Biomedical Research, vol 10, pp. 459-470, 1977.

9. Afonso VX, Tompkins WJ, Nguyen TQ, Trautmann S, and Luo S: Filter bank-based processing of the stress ECG. Proc. Annu. Int. conf. IEEE Eng. Med. Biol. Soc., Sep. 1995.

10. Albrecht P, Rice K, Jarisch WR, and Mark G: Efficient measurement of long term ST segment trends. Proc. of the Fifth Annu. Conf., pp. 644-649, 1983

11. Vaidyanathan PP: Multirate systems and filter banks. Englewood Cliffs, NJ. Prentice-Hall, 1993.

12. Queiroz RL, Nguyen TQ, and Rao KR: The class of GenLOTs: Generalized linear-phase lapped orthogonal transforms. Proc. ISCAS. no. 2 , 277-280, 1994

13. Soman AK, Vaidyanathan PP, and Nguyen TQ: Linear phase paraunitary filter banks: Theory, factorizations and designs. IEEE Trans. on Signal Processing, vol. 41, no. 12., pp. 3480-3495, 1993.

14. Malvar HS: Signal processing with lapped 15. Weisner SJ, Tompkins WJ, and Tompkins of the committee of electrocardiography and cartransforms, Artech House, Norwood, MA, 1992. 
BM: A compact, microprocessor-based ECG STsegment monitor for the operating room IEEE Trans. Biomed Eng., BME-29 (9):642 649, 1982 16. MIT/BIH Database Distribution, Massachusetts Inst: Technol., 77 Massachusetts Avenue, Room 20A-113, Cambridge, MA 02139.

17. Luo S, and Tompkins WJ: QRS complex alignment for averaging of cardiac signals. Joum. of Electrocardiol., 1994

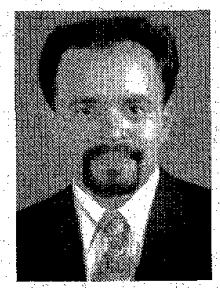

Valtino $X$. Afonso (S'91) received the B.S. and M.S. degrees in electrical and computer engineering from Marquette University, Milwaukee, WI., in 1990 , and the University of Wisconsin, Madison, WI, in 1993 , respectively. He is presently doing graduate work at the University of Wisconsin, Madison, and is pursuing the $\mathrm{Ph} . \mathrm{D}$ degree His research interests in clude the application of signal proccesing concepts such as time-frequency analysis, wavelets, multirate processing, filter bank-based algorithms, and classification algorithms to biomedical and image processing solutions.

Afonso is a contributing author to $D e$ sign of pacemakers and implantable cardioverter-defibrillators, (J. G. Webster, Ed., Piscataway, NJ: IEEE Press, 1995), and Biomedical Digital Signal Processing: C-Language Examples and Laboratory Experiments for the $I B M P C$, (W. J.Tompkins, Ed, Englewood Cliffs, NJ: Prentice-Hall, 1993). He is a member of Tau Beta Pi

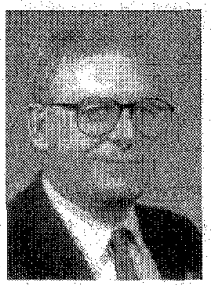

Willis J. Tompkins (S'61-M'66-SM'77-F

'92) received the B.S. and M.S. degrees in electrical engineering from the University of Maine, Orono, in 1963 and 1965 , respectively and the Ph.D. degree in biomedical electronic engineering from the University of Pennsylvania, Philadel phia, in 1973.

From 1965 to 1968 , he was an electrical engineer at Sanders Associates, Inc. Nashua, $\mathrm{NH}$, where he worked on research and development of data storage systems. He was employed from 1973 to 1974 at the Hospital of the University of Pennsylvania as a biomedical engineer. Since 1974, he has been on the faculty of the University of Wisconsin-Madison.

Currently, he is Professor and Chair of the Department of Electrical and Com- puter Engineering. He teaches undergraduate, graduate, and short courses on the topic of computers in medicine. His research interests include applications of microcomputer-based medical instrumentation and on-line biomedical computing, $\mathrm{He}$ is editor of the Prentice Hall textbook, Biomedical Digital Signal Processing C Language Examples and Laboratory Experiments for the $\mathrm{IBM} P \mathrm{PC}, 1993$. $\mathrm{He}$ is coeditor with $\mathrm{I}$. $\mathrm{G}$. Webster of the Prentice-Hall textbooks, Design of Microcomputer-Based Medical Instrumentation, 1981, and Interfacing Sensors to the IBM PC, 1988. He is coeditor with J. G. Webster, A. M. Cook, and G. C. Vanderheiden of the Chapnan Hall textbook, Electronic Devices for Rehabilitation, 1985.

Dr. Tompkins is a past President of the IEEE Engineering in Medicine and Biology Society. He is also a member of the IEEE Computer Society, the Association for the Advancenent of Medical Instrumentation, the Biomedical Engineering Society, and the American Society for Engineering Education. He is a Fellow of the IEEE and the American Institute for Medical and Biological Engineering. He is a Registered Professional Engineer in Wisconsin. Address for correspondence: Professor Willis I. Tompkins, Department of Electrical and Comaputer Engineering, University of Wiscoisin, 1415 Engineering Drive, Madison, WI 53706 .

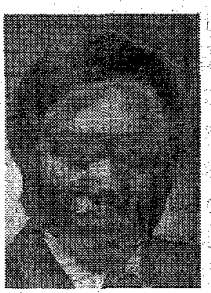

Truong $Q$ Nguyen re ceived the B. S., M. S. and Ph.D. degrees in electrical engineering from the Californa $\mathrm{In}$ stitute of Technology, Pasadena, in 1985 , 1986 , and 1989 , respectively. He was with MIT Lincoln laboratory from June 1989 to July 1994, as a member of technical staff. During the academic yeat 1993-1994, he was a visiting lecturer at MIT and an adjunct professor at Northeastern University Since August 1994 he bas been at the University of Wisconsin Madison, where he is currently an assistant professor of electrical and computer engineering. His research interests are in digital and image signal processing, multirate systems, wavelets and applications biomedical signal processing, filter design, and ultrasonics nondestructive evaluation.

Prof. Nguyen was a recipient of a fellowship from Aerojet Dynamics for advanced studies. He received the IEEE
Transaction in Signal Processing Paper Award (Image and Multidimensional Processing area) for the paper he co wrote with Prof P,P Vaidyanathan on linearphase perfect-reconstruction filter bakks (1992). He reeelved the NSF Career Award in 1995 and is the coauthor (with Prof Gilbert Strang) of a texibook on Wavelets and Filter Banks Cambridge Wellesley Press. Ho is currently an Assocrate Editor for the IEEE Transaction on SignalProcessing and is also served in the DSP Technical Connittee for CAS society. He is a member of Tau Beta, Di, Eta Kappa Nu and Sigma XI.

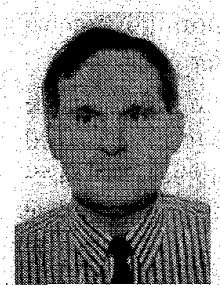

Kurt Michler (M75) is an independent medical device consultant PreMionsly he was Vice President of Technology at Burdick, Inc. He holds a BS and an MS degree in Electrical Engineering fom the University of Wisconsin and has 20 years experience in the design of medical instu ments, nost of it in the areas of electro cardiography, metabolie measurements and pulmonary function testing. His pri mary interests include the development and testing of neasurement and analysis software Michler is a member of A AMI and the IEEEMEM and Conputer Societies. tht

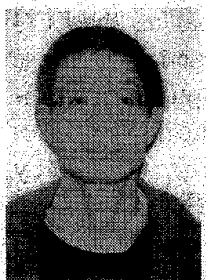

Shen Luo teceived the B S. degree in electrieal engineering and MS degree in bionedical engineering from Huazhong University of Selence and Technol ogy, Wuhan, China, in 1982 and 1985 , respec tively, and the $\mathrm{PhD}$ degree in electrical engineering from the Univer sity of Wis consin-Madison, Wisconsin, in 1993 . He is currently a tesearch engineer at Burdick, Inc. Milton, Wisconsin, where he is working on ECG signal processing and detection algorithms. Dr. Luo has published articles in the areas of bighresolution $E C G$, heart rate variability, ECG detection, nolse reduction for bioele trode neas urements, ECG to VCG transformation, ECG monitoring the electrode system in impedance based ventila tion menitoring, and detection techniques for ventilation monitoring $\mathrm{He}$ is a contrib uting author to Prevention of Pressure Sores Engineering and Clinical Aspects (Bristel, England: Adam Hilger, 1991) 